\title{
Influence of negative lymph node in No 7 on survival of patients with middle thoracic esophageal squamous cell carcinoma
}

\author{
Jinling Zhang' \\ Xueyuan Heng' \\ Yi Luo ${ }^{2}$ \\ Qingxi Fu' \\ Zhengrong $\mathrm{Li}^{\prime}$ \\ Fengyuan Che' \\ Baosheng $\mathrm{Li}^{3}$ \\ 'Cancer Center, LinYi People Hospital, \\ Affiliated to Shandong University, \\ School of Medicine, Jinan, Shandong \\ Province, People's Republic of \\ China; ${ }^{2}$ Department of Radiation \\ Oncology, University of Michigan, \\ Ann Arbor, MI, USA; ${ }^{3}$ Department \\ of Radiation Oncology (Chest \\ Section), Shandong's Key Laboratory \\ of Radiation Oncology, Shandong \\ Cancer Hospital, Shandong Academy \\ of Medical Sciences, Jinan, Shandong \\ Province, People's Republic of China
}

Correspondence: Fengyuan Che LinYi People Hospital, Affiliated to Shandong University, School of Medicine, No 44 Wenhua Xi Road, Jinan 2500I2, Shandong Province, People's Republic of China

Tel +86539807 2679

Fax +865398072679

Email che1971@126.com

Baosheng Li

Shandong Cancer Hospital, Shandong Academy of Medical Sciences, No 440 Jiyan Road, Jinan 250I I7, Shandong Province, People's Republic of China Tel +8653167626161

Fax +86 53I 6762 6I6I

Email baoshli1963@163.com
This article was published in the following Dove Press journal:

OncoTargets and Therapy

30 March 2016

Number of times this article has been viewed

Background: The overall survival (OS) of patients with thoracic esophageal cancer is poor because of the high rate of lymph node metastases. However, recent studies found that the negative lymph node (LN) may also influence the patients' OS. The purpose of this study is to investigate which negative LN stations play a key role in OS prediction.

Method: Our study included the retrospective records of 99 patients, who were identified with middle thoracic esophageal squamous cell cancer after esophagectomy. The maximum follow-up time was 6 years. Cox regression models were employed to determine the association between the negative LN and OS of patients. After applying Kaplan-Meier method to calculate OS of patients with positive and negative LNs, the log-rank tests were used to assess the difference between them.

Result: The hazard ratio of the total number of negative LNs was 0.937 ( $P=0.001)$, and the length of tumor was $1.166(P=0.038)$. Multivariate regression results showed that the numbers of positive LNs in No 3 and 7 stations and negative LNs in No 109 and 7 stations were significantly related to OS, and their $P$-values were $0.017,0.001,0.020$, and 0.022 , respectively. The OS of the patients who had positive and negative LNs in No 7 station was significantly different $(P=0.028)$.

Conclusion: No 7 is the most important among the negative LN stations which prolong OS. More attention should be paid to this area when making treatment plan for patients with no negative LNs identified in operation.

Keywords: esophageal cancer, lymph node metastasis, prognosis

\section{Introduction}

Esophageal cancer (EC) is prevalent in Asia, and the high rate of lymph node metastasis (LNM) influences the survival of the patients. ${ }^{1,2}$ Chemo-radiotherapy is the main method to prolong OS after patients experienced the esophagectomy and lymphadenectomy.

Radiotherapy (RT) plays a key role in controlling local recurrence of the tumor that is a major cause of treatment failure. The treatment plan is made on the basis of the pattern of LNMs with pathologically positive lymph node (LN), but some researchers recently reported that the negative LN also has a close relationship with the outcome. Greenstein et al found that the number of LNs negatively affected the postoperative survival based on the Surveillance, Epidemiology, and End Results (SEER) cancer data. ${ }^{3}$ Zhang et al, Jang et al, and Smith et al proposed that greater counts of total negative LNs were associated with longer survival of EC patients. ${ }^{1,2,4}$ 
Nevertheless, which station of negative LN will significantly influence the outcome of EC patients has not been investigated to the best of our knowledge. Although surgeons try to resect more LNs, the 5-year overall survival (OS) rate of patients with EC is still 16\% ${ }^{1,2,5}$ Some scholars clarified that the region of LNM was more important than the number of LNMs. ${ }^{6,7}$

Researchers reported that the No 107, 108, and 7 stations of LNs were the commonest stations of metastases, and they were all included in the treatment planning. ${ }^{7}$ But the dose received in No 7 was $<50$ Gy in most cases for the middle thoracic EC. The limited dose might be one of the reasons for tumor local occurrence.

Furthermore, because the negative $\mathrm{LN}$ is closely related to OS and its importance is frequently neglected in treatment planning, it is necessary to investigate which negative LN station is closely related to the outcome. Since middle thoracic esophageal squamous cell carcinoma (ESCC) is the major histological type of EC, we limit the inclusion criteria only to middle thoracic ESCC in order to avoid the influence of confounders of pathology type and site of lesion.

\section{Materials and methods Study population}

The retrospective information of 99 patients with ESCC at the Department of Oncology in LinYi People Hospital, affiliated to the Shandong University, People's Republic of China, between January 2008 and February 2009, was collected. All patients underwent curative esophagectomy with systemic lymphadenectomy. Only the patients with middle thoracic tumor and Stage II/III tumor are included in our study, and tumor stage is assessed according to the criteria of Union for International Cancer Control (2002 edition). The patients who satisfy the following criteria are excluded from our study: 1) underwent preoperative RT or chemotherapy, 2) not identified with ESCC by pathologist, and 3) less than ten LNs were collected in operation.

Upper gastrointestinal endoscopy, computed tomography of the chest and abdomen, and ultrasound of the cervical region were performed to examine the possibility of LNM before operation. Seventy-two patients underwent two-field LN dissection, and 25 patients received three-field LN dissection because of the possible metastasis in clavicle area.

All LNs were collected by surgeons at the end of the operation and analyzed independently by two pathologists. The study has been approved by the Research Ethics Committee of LinYi People Hospital. Written informed consent were obtained from each patient.

\section{Follow-up and statistical analysis}

All patients were followed up every 3 months over the first 2 years and every 6 months thereafter, until the maximum follow-up time of 6 years or the date of death came. The patients' OS was calculated from the date of operation until death or last follow-up time. Phone calls were performed to learn the status of patients.

We used $\chi^{2}$ test to evaluate the relationship between the clinic pathological factor and the LNM. The difference between the distribution pattern of positive and negative LN in every station was evaluated by Wilcoxon rank sum test. Cox proportional hazards model was used to analyze the relation between the LN station and the survival, and a forward stepwise elimination with a threshold of $P=0.1$ was used to select parameters. Survival analysis was conducted by employing Kaplan-Meier method, and the log-rank test was used to compare the difference in survival between different stations. For a two-sided test, the relationship with $P$-value $<0.05$ was considered statistically significant. Moreover, all the data were analyzed by using SPSS version 17.0 (SPSS Inc., Chicago, IL, USA).

\section{Results}

\section{Patient demographics and OS}

The characteristics of 99 patients are described in Table 1. The average total number of LNs in each patient is 15.0, and the mean number of negative LNs is 13.5. The median follow-up is 36 months (range: 1-72 months), and the 5-year survival rate is $31.0 \%$.

The tumor length, depth of tumor invasion, pathological differentiation, and postoperative therapy all had significant relationships with the positive status of $\mathrm{LN}$, and their $P$-values are $0.005,0.006,0.007$, and 0.001 , respectively. Nevertheless, none of the earlier parameters is identified to be closely related to the number of negative LNs.

To accurately describe the incidence and pattern of LNM, the terminology of the regional LNs of ESCC follows the definition from the Japanese Society for Esophageal Diseases.

\section{The pattern of positive and negative LN in LN stations}

The difference between the distribution pattern of positive and negative LN in every station is significant, since the $P$-value is equal to 0.018 (Table 2). In the meantime, the percentages of positive $\mathrm{LN}$ in $\mathrm{LN}$ stations are similar to the results of our past work. ${ }^{8}$ The percentages of negative LNs in stations $108,107,7,3,2$, and 109 were $25.8 \%, 24.5 \%$, $26.7 \%, 0.5 \%, 5.6 \%$, and $3.4 \%$, respectively. 
Table I The main clinical and pathological variables of the patients with pathological positive lymph node

\begin{tabular}{|c|c|c|c|c|c|}
\hline Characteristics & Total number of LNs & Number of positive LNs & $P$-value & Number of negative LNs & $P_{\text {-value }}{ }^{a}$ \\
\hline \multicolumn{6}{|l|}{ Age (years) } \\
\hline$<60$ & 505 & 59 & 0.086 & 446 & 0.668 \\
\hline$\geq 60$ & 976 & 84 & & 892 & \\
\hline \multicolumn{6}{|l|}{ Sex } \\
\hline Male & 1,202 & 125 & 0.212 & 1,119 & 0.76 \\
\hline Female & 223 & 16 & & 207 & \\
\hline \multicolumn{6}{|c|}{ Length of tumor $(\mathrm{cm})$} \\
\hline$\leq 4$ & 643 & 42 & 0.005 & 601 & 0.719 \\
\hline $4-6$ & 468 & 54 & & 414 & \\
\hline$\geq 6$ & 370 & 47 & & 323 & \\
\hline \multicolumn{6}{|l|}{ Differentiation } \\
\hline Well & 651 & 44 & 0.006 & 607 & 0.732 \\
\hline Moderate & 702 & 87 & & 615 & \\
\hline Poor & 128 & 12 & & 116 & \\
\hline \multicolumn{6}{|c|}{ Depth of tumor invasion } \\
\hline TI-T2 & 91 & 1 & 0.007 & 90 & 0.529 \\
\hline $\mathrm{T} 3-\mathrm{T} 4$ & 1,390 & 142 & & 1,248 & \\
\hline \multicolumn{6}{|c|}{ Postoperative therapy } \\
\hline Yes & 878 & 105 & 0.001 & 773 & 0.416 \\
\hline No & 603 & 38 & & 565 & \\
\hline
\end{tabular}

Note: a From a chi-square test.

Abbreviation: LN, lymph node.

\section{Impact of total number of negative LNs on survival}

In terms of the relationship with OS, the total number of negative LNs is more significant than any other parameters. The hazard ratio (HR) is $0.937,95 \%$ confidence interval (CI) is $0.902-0.973$, and the $P$-value is 0.001 (Table 3). The OS between more than and less than five negative LNs identified after operation was significantly different, with $P=0.0045$. Similar results can be obtained for the threshold number of ten and 15 negative LNs identified after operation, since the $P$-values are 0.0002 and 0.0015 , respectively, as shown in Figure 1.

\section{Impact of $L N$ stations on survival}

Among negative LN stations, No 109 and No 7 have significant relation with the OS, and the HR was 0.600 (95\% CI $0.391-0.920, P=0.019)$ and 0.921 (95\% CI 0.855-0.993,

Table 2 The distribution of pathological positive and negative LN

\begin{tabular}{llll}
\hline LN stations & Positive LN & Negative LN & P-value $^{\mathbf{a}}$ \\
\hline 108 & $57(39.8 \%)$ & $343(25.8 \%)$ & \\
107 & $23(16.1 \%)$ & $326(24.5 \%)$ & \\
7 & $44(30.8 \%)$ & $355(26.7 \%)$ & \\
3 & $2(1.4 \%)$ & $6(0.5 \%)$ & \\
2 & $8(5.6 \%)$ & $75(5.6 \%)$ & \\
109 & $1(0.7 \%)$ & $45(3.4 \%)$ & \\
Other location & $8(5.6 \%)$ & $179(13.4 \%)$ & \\
Statistical differences & & & 0.018 \\
\hline
\end{tabular}

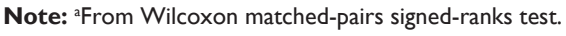
Abbreviation: LN, lymph node.
$P=0.033$ ), respectively. Among positive LN stations, No 3 and No 7 have a close relationship with the OS (HR 9.677, 95\% CI 1.438-65.117, $P=0.02$; HR 1.510, 95\% CI 1.022 2.231, $P=0.038$ ) (Table 4).

Log-rank test revealed that the OS between the patients who have only negative LNs and have positive LNs in No 7 station is significantly different $(P=0.028)$. Significant difference is not identified between the patients who have only negative LNs and have positive LNs in No 109 station, but different trends emerge within 36-month follow-up period (Figure 2). The anatomic locations of No 109 and No 7 LN stations in treatment planning are shown in Figure 3.

\section{Discussion}

The OS of 5 years is still poor for the thoracic ESCC, especially in late-phase patients. The major reason of relapse is the high rate of LNMs, which is a big challenge for

Table 3 The relationship between the total number of positive and negative LNs and OS

\begin{tabular}{llllll}
\hline Factor & $\begin{array}{l}\text { Hazard } \\
\text { ratio }\end{array}$ & $\begin{array}{l}\text { Standard } \\
\text { error }\end{array}$ & $\boldsymbol{Z}$ & $\mathbf{P}>\mathbf{z}$ & $\begin{array}{l}\mathbf{9 5 \%} \text { confidence } \\
\text { interval }\end{array}$ \\
\hline Length & $\mathrm{I} .138$ & 0.089 & $\mathrm{I} .650$ & 0.099 & $0.976-1.327$ \\
Male & 0.784 & 0.277 & -0.690 & 0.490 & $0.392-1.567$ \\
pt & 1.075 & 0.063 & 1.240 & 0.215 & $0.959-1.205$ \\
nt & 0.937 & 0.018 & -3.380 & 0.001 & $0.902-0.973$ \\
\hline
\end{tabular}

Abbreviations: LN, lymph node; OS, overall survival; pt, total number of positive $\mathrm{LNs}$; nt, total number of negative LNs. 

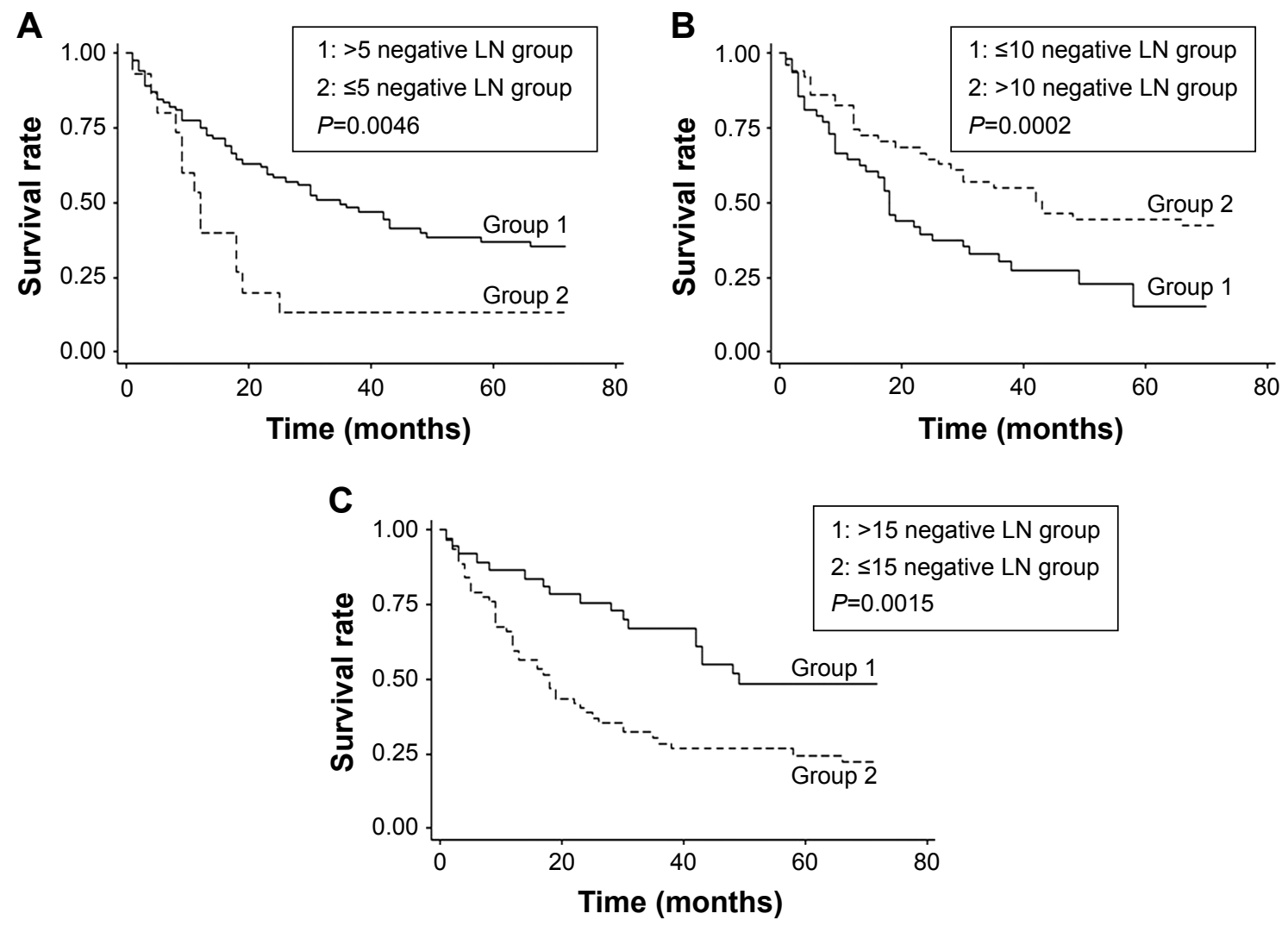

Figure I The survival difference of patients with different total negative LNs.

Notes: (A) The OS of patients with more than and less than five total number of negative LNs. Group I: patients with more than five negative LNs in operation. Group 2: patients with less than five negative LNs. The difference between them was significant $(P=0.0045)$. (B) The OS of patients with more than and less than ten negative $L N s$. Group I: patients with less than ten negative LNs. Group 2: patients with more than ten negative LNs. The difference between them was significant ( $P=0.0002)$. (C) The OS of patients with $>15$ and $<15$ total number of negative LNs. Group I: patients with $>15$ negative LNs. Group 2: patients with $<15$ negative LNs. The difference between them was significant $(P=0.0015)$.

Abbreviations: OS, overall survival; LN, lymph node.

lymphadenectomy in operation. ${ }^{1,2}$ Postoperative RT in the high-risk area of LNMs can enhance the long-term survival in ESCC. ${ }^{1,2,8}$

Involved-field irradiation is applied in the target planning of postoperative RT in ESCC. ${ }^{9,10}$ It refers to the pathological positive area of $\mathrm{LN}$ which is considered as being closely related to the outcome of patients. ${ }^{11-13}$ But there is still

Table 4 The relationship between the total negative LN number of every station and OS

\begin{tabular}{llllll}
\hline Factor & $\begin{array}{l}\text { Hazard } \\
\text { ratio }\end{array}$ & $\begin{array}{l}\text { Standard } \\
\text { error }\end{array}$ & $\boldsymbol{Z}$ & $\mathbf{P}>\mathbf{z}$ & $\begin{array}{l}\text { 95\% confidence } \\
\text { interval }\end{array}$ \\
\hline Male & 0.642 & 0.235 & -1.21 & 0.226 & $0.313-1.315$ \\
P2 & 0.567 & 0.243 & -1.32 & 0.186 & $0.245-1.314$ \\
P3 & 9.677 & 9.413 & 2.33 & 0.02 & $1.438-65.117$ \\
P7 & 1.510 & 0.301 & 2.07 & 0.038 & $1.022-2.231$ \\
n106 & 0.528 & 0.233 & -1.45 & 0.147 & $0.222-1.253$ \\
n107 & 0.946 & 0.033 & -1.58 & 0.114 & $0.882-1.013$ \\
n109 & 0.600 & 0.131 & -2.34 & 0.019 & $0.391-0.920$ \\
n7 & 0.921 & 0.035 & -2.14 & 0.033 & $0.855-0.993$ \\
\hline
\end{tabular}

Abbreviations: $L N s$, lymph nodes; OS, overall survival; $p$, positive $L N$; $n$, negative $\mathrm{LN}$. controversy about which LN stations should be included in treatment planning. ${ }^{14-17}$ The LN stations of 101, 104-R, 105, 106rec-L, 106tb-L, 106tb-R, 107, 108, 110, 112, 2, 3 , and 7 are generally considered as high-risk regions and are commonly included in treatment planning in thoracic ESCC. ${ }^{18}$

But recently, researchers found that pathological negative LN could also influence outcome of patients. ${ }^{19-21}$ Zhu et al found that the total number of negative LNs was associated with the survival of patients undergoing three-field lymphadenectomy. ${ }^{22}$ Greenstein et al also found that the number of negative LNs had relation with the postoperative survival based on the SEER cancer data, which is a nationally representative, population-based cancer record. ${ }^{3}$ Smith et al reported that more negative LNs in operation led to longer survival. ${ }^{4}$

The earlier result suggested that the distribution of negative LN in LN stations is important, and it is necessary to investigate which negative LN stations have significant relationship with OS. The distribution and the number of negative LNs could reflect the range of esophagectomy, 

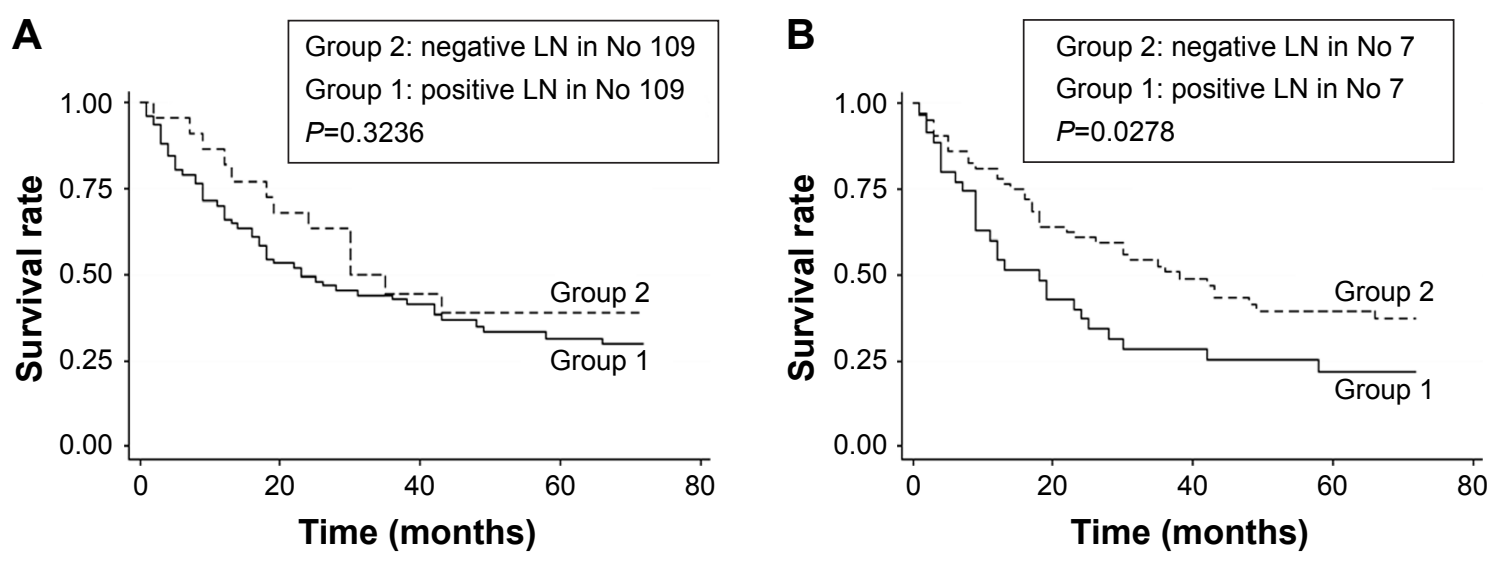

Figure 2 The survival difference of patients with negative and positive LN

Notes: (A) The OS of patients with negative and positive lymph nodes in No 109. Group I: patients with negative lymph nodes in No 109. Group 2: patients with positive lymph nodes in No 109 ( $P=0.3235)$. (B) The OS of patients with negative and positive lymph nodes in No 7. Group I: patients with positive lymph nodes in No 7. Group 2: patients with negative lymph nodes in No 7. The difference between them was obvious $(P=0.0275)$.

Abbreviations: OS, overall survival; LN, lymph node; No, number.

so it should be used as a reference for treatment planning decision-making in postoperation irradiation.

In this study, the length of tumor, the differentiation, and depth of tumor invasion had significant relationship with the rate of positive $\mathrm{LN}$ (the $P$-values are all $<0.001$ ). The rates of No 108,107 , and 109 LN stations are $21.1 \%, 27.9 \%$, and $4.7 \%$, respectively, and these results are similar to previously published papers. ${ }^{19,20}$ But the rate of negative LNs in every stations has not been reported, and there is significant difference between the distribution of positive and negative LNs in LN stations $(P=0.0001)$. It might be implied that it is a big challenge for the surgeon to resect LNM entirely in all LN stations by considering the different anatomical structure of LN stations.

In this study, the total number of negative LNs influenced outcome significantly. This result is similar to the conclusions of previously published articles. Significant relation between the total number of positive LNs and prognosis was not confirmed in this study, which might be weakened by the robust relation between the total number of negative LNs and prognosis in this Cox regression model analysis. In addition, obvious differences of OS have been identified between the following groups: patients with more than and less than five negative LNs identified after operation $(P=0.004)$, patients with more than and less than ten negative LNs $(P=0.0002)$, and patients with $>15$ and $<15$ negative $\operatorname{LNs}(P=0.001)$. Zhu et al also proved the similar result of negative $\mathrm{LNs}$ but failed to find a threshold for negative LNs to resect in operation, where patients could be benefited from it. ${ }^{22}$ The possible reason could be that it was very hard to find a balance point between the number of LNs resected and the injury due to operation.

As far as we know, negative LN stations that are able to significantly influence the outcome are not reported. ${ }^{1,2,23-26}$ Based on the result of this study, 7 and 109 stations were significantly associated with outcome. The possible reason might be that 7 and 109 stations are all at the edge of the treatment planning in postoperation radiation as shown in Figure 3, and they received low dose (No 7) or were completely outside the area of treatment planning (No 109). Another reason might be that the locations of No 7 and 109 are more complicated than any other LN stations in anatomy
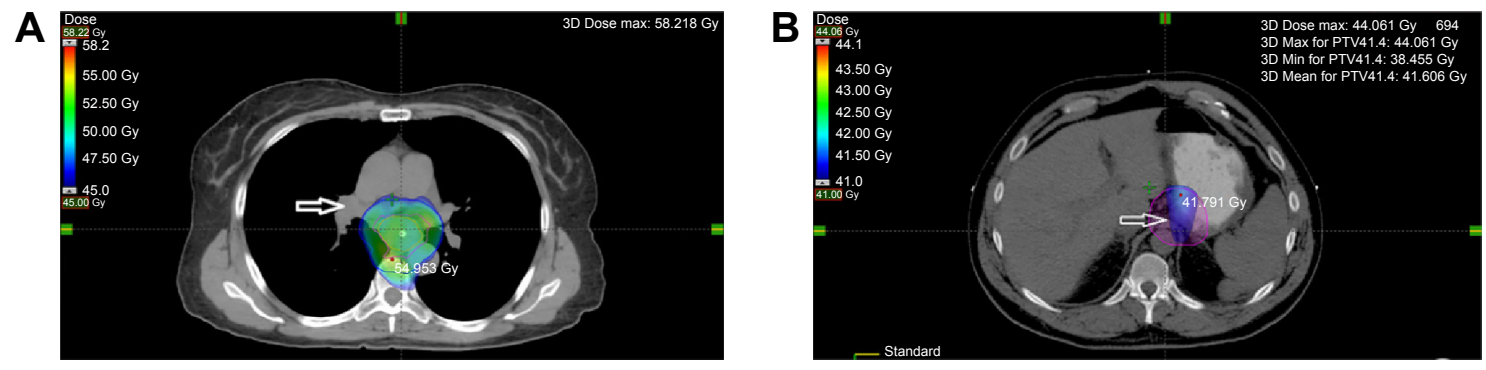

Figure 3 The anatomical site of (A) No 109 and (B) No 7 in treatment planning.

Note: The white arrow shows the anatomical site of No 109 (A) and No 7 (B), and the former was outside the treatment planning of RT while the latter was inside the treatment planning of RT. 
and are hard to resect absolutely in operation, which lead to false negative. Another result in this study shows that the OS between the patients who have positive and have only negative LN in 7 station is different $(P=0.028)$. Although similar result is not identified in 109 station $(P=0.324)$, OS rate is significantly different during initial 36 months after operation. This result further indicates that the negative LN in 7 stations could influence the outcome significantly. Because the LN metastasis frequency in No 109 is low and has a little effect on the protocol of treatment planning in RT, the influence of No 109 on OS should be investigated in future research on a large scale. If only positive LNs are removed from 7 station, it possibly implies that positive LN is not thoroughly resected and other positive LNs are missed because of the complicated anatomical structure of this LN station. On the contrary, if negative LNs are identified in this LN station, it supports that there are no positive LNs left. More negative LNs might mean lower possibility of LNM in this LN station, which is supported by the earlier analysis of Cox regression models. Therefore, we should pay more attention to station 7 in treatment planning of postoperation irradiation if no negative $\mathrm{LN}$ in this area is identified in operation..$^{9,18,27-30}$

Limitations of this study are as follows. First, although the inclusion of this study is strict and only the middle thoracic ESCC is collected, it is a retrospective study and is subject to inherent biases. Second, although we limit the inclusion, confounders are still unavoidable because it is a small-scale study. Third, the representativeness of this study might be limited because it is a single-center research, and the result could be influenced by the protocol of esophagectomy in our hospital.

In summary, results in this study show that the negative LN in 7 station could influence the outcome significantly, and patients with no negative LN identified in this LN station in operation might be benefited from elective nodal irradiation of this LN station or can be given more doses of this irradiation.

\section{Acknowledgment}

This study was supported by Shandong Provincial Medical and Health Development Plan (2013WSA13018) and Natural Science Foundation of Shandong Province (ZR2014HL062).

\section{Disclosure}

The authors report no conflicts of interest in this work.

\section{References}

1. Zhang HL, Chen LQ, Liu RL, et al. The number of lymph node metastases influences survival and international union against cancer tumornode-metastasis classification for esophageal squamous cell carcinoma. Dis Esophagus. 2010;23(1):53-58.

2. Jang HJ, Lee HS, Kim MS, Lee JM, Zo JI. Patterns of lymph node metastasis and survival for upper esophageal squamous cell carcinoma. Ann Thorac Surg. 2011;92(3):1091-1097.

3. Greenstein AJ, Litle VR, Swanson SJ, Divino CM, Packer S, Wisnivesky JP. Effect of the number of lymph nodes sampled on postoperative survival of lymph node-negative esophageal cancer. Cancer. 2008;112(6):1239-1246.

4. Smith D, Schwarz R, Schwarz R. Impact of total lymph node count on staging and survival after gastrectomy for gastric cancer: data from a large US-population database. J Clin Oncol. 2005;23(28):7114-7124.

5. Li Q, Wu S, Gao J, Xu J, Hu L, Xu T. Impact of esophageal cancer staging on overall survival and disease-free survival based on the 2010 AJCC classification by lymph nodes. J Radiat Res. 2013;54(2):307-314.

6. Chen J, Pan J, Zheng X, et al. Number and location of positive nodes, postoperative radiotherapy, and survival after esophagectomy with three-field lymph node dissection for thoracic esophageal squamous cell carcinoma. Int J Radiat Oncol Biol Phys. 2012;82(1):475-482.

7. de Meer S, Dauwan M, de Keizer B, Valk G, Rinkes I, Vriens M. Not the number but the location of lymph nodes matters for recurrence rate and disease-free survival in patients with differentiated thyroid cancer. World J Surg. 2012;36(6):1262-1267.

8. Lu JC, Kong C, Tao H. Radiotherapy with or without concurrent chemotherapy for lymph node recurrence after radical surgery of thoracic esophageal squamous cell carcinoma. Int J Radiat Oncol Biol Phys. 2010;78(3):710-714.

9. Li M, Zhang X, Liao Z, et al. Failure patterns after definitive chemoradiation therapy with involved-field irradiation for locally advanced esophageal squamous cell carcinoma. Int $J$ Radiat Oncol Biol Phys. 2014;2014(90):S12-S12.

10. Zhang X, Li M, Meng X, et al. Involved-field irradiation in definitive chemoradiotherapy for locally advanced esophageal squamous cell carcinoma. Radiat Oncol. 2014;2014:9.

11. Lazarescu I, Thureau S, Nkhali L, Pradier O, Dubray B. [Clinical target volume delineation for radiotherapy of the esophagus]. Définition $d u$ volume cible anatomoclinique pour l'irradiation des cancers de l'oesophage. Cancer Radiother. 2013;17(5-6):453-460.

12. Song Y, Liang Y, Zang R, Hu L, Zhu S. Application of serial section method to determine the radiotherapy target volume for esophageal squamous carcinoma. Cell Biochem Biophys. 2013;66(2):351-356.

13. Wang ZW, Zhang W, Dong W, et al. Pathological analysis of extracapsular extension of metastatic lymph node and its potential impact on nodal clinical target volume in the radiotherapy of esophageal squamous cell carcinoma. Neoplasma. 2014;61(3):324-330.

14. Jingu K, Ariga H, Nemoto K, et al. Long-term results of radiochemotherapy for solitary lymph node metastasis after curative resection of esophageal cancer. Int J Radiat Oncol Biol Phys. 2012;83(1):172-177.

15. Xu Y, Liu J, Du X, et al. Prognostic impact of postoperative radiation in patients undergoing radical esophagectomy for pathologic lymph node positive esophageal cancer. Radiat Oncol. 2013;2013:8.

16. Shridhar R, Weber J, Hoffe S, Almhanna K, Karl R, Meredith K. Adjuvant radiation therapy and lymphadenectomy in esophageal cancer: a SEER database analysis. J Gastrointest Surg. 2013;17(8):1339-1345.

17. Liu Q, Cai X, Wu B, Yu W, Zhao K, Fu X. Characteristics of lymph node recurrence after radical surgery and their value in target volume delineation in postoperative radiation therapy for thoracic esophageal squamous cell carcinoma. Int J Radiat Oncol Biol Phys. 2013;87(2):S298-S298.

18. Ji K, Zhao L, Yang C, Meng M, Wang P. Three-dimensional conformal radiation for esophageal squamous cell carcinoma with involved-field irradiation may deliver considerable doses of incidental nodal irradiation. Radiat Oncol. 2012;2012:7. 
19. Cheng J, Kong L, Huang W, et al. Explore the radiotherapeutic clinical target volume delineation for thoracic esophageal squamous cell carcinoma from the pattern of lymphatic metastases. J Thorac Oncol. 2013; 8(3):359-365.

20. Huang W, Li B, Gong H, et al. Pattern of lymph node metastases and its implication in radiotherapeutic clinical target volume in patients with thoracic esophageal squamous cell carcinoma: a report of 1077 cases. Radiother Oncol. 2010;95(2):229-233.

21. MacGuill MJ, Barrett C, Ravi N, MacDonald G, Reynolds JV. Isolated tumour cells in pathological node-negative lymph nodes adversely affect prognosis in cancer of the oesophagus or oesophagogastric junction. J Clin Pathol. 2007;60(10):1108-1111.

22. Zhu Z, Chen $\mathrm{H}, \mathrm{Yu}$ W, et al. Number of negative lymph nodes is associated with survival in thoracic esophageal squamous cell carcinoma patients undergoing three-field lymphadenectomy. Ann Surg Oncol. 2014; 21(9):2857-2863.

23. Feng JF, Zhao Q, Chen QX. Prognostic value of subcarinal lymph node metastasis in patients with esophageal squamous cell carcinoma. Asian Pac J Cancer Prev. 2013;14(5):3183-3186.

24. Tanaka H, Ohira M, Kubo N, et al. Association of location of lymph node metastases with postoperative recurrence of esophageal squamous cell carcinoma. Anticancer Res. 2012;32(8):3421-3426.
25. Hsu PK, Huang CS, Hsieh CC, Wu YC, Hsu WH. Role of right upper mediastinal lymph node metastasis in patients with esophageal squamous cell carcinoma after tri-incisional esophagectomies. Surgery. 2014.

26. Li H, Yang S, Xiang J, Chen H. The number of lymph node metastases influences survival and International Union Against Cancer tumor-nodemetastasis classification for esophageal squamous cell carcinoma: does lymph node yield matter? Dis Esophagus. 2011;24(2):108.

27. Liu Q, Cai X, Wu B, Zhu Z, Chen H, Fu X. Patterns of failure after radical surgery among patients with thoracic esophageal squamous cell carcinoma: implications for the clinical target volume design of postoperative radiotherapy. PLoS One. 2014;9(5):e97225.

28. Zhang X, Li M, Kong L, Yu J. Involved field irradiation compared with prophylactic nodal irradiation to unresectable esophageal carcinoma: a retrospective study. J Clin Oncol. 2013;31(15).

29. Kawaguchi Y, Nishiyama K, Miyagi K, Suzuki O, Ito Y, Nakamura S Patterns of failure associated with involved field radiotherapy in patients with clinical stage I thoracic esophageal cancer. Jpn J Clin Oncol. 2011; 41(8):1007-1012.

30. Huang Y, Feng JF. Low preoperative lymphocyte to monocyte ratio predicts poor cancer-specific survival in patients with esophageal squamous cell carcinoma. OncoTargets Ther. 2015;8:137-145.
OncoTargets and Therapy

\section{Publish your work in this journal}

OncoTargets and Therapy is an international, peer-reviewed, open access journal focusing on the pathological basis of all cancers, potential targets for therapy and treatment protocols employed to improve the management of cancer patients. The journal also focuses on the impact of management programs and new therapeutic agents and protocols on

\section{Dovepress}

patient perspectives such as quality of life, adherence and satisfaction. The manuscript management system is completely online and includes a very quick and fair peer-review system, which is all easy to use. Visit http://www.dovepress.com/testimonials.php to read real quotes from published authors. 